\title{
X-ray absorption study of the electronic structure of Mn-doped amorphous Si
}

\author{
Li Zeng (曾立), ${ }^{*}$ A. Huegel, E. Helgren, and F. Hellman \\ Department of Physics, University of California, Berkeley, Berkeley, CA, 94720, USA \\ C. Piamonteze and E. Arenholz \\ Lawrence Berkeley National Lab, Advanced Light Source, Berkeley, CA, 94720, USA
}

(Dated: March 8, 2008)

\begin{abstract}
The electronic structure of $\mathrm{Mn}$ in amorphous $\mathrm{Si}\left(a-\mathrm{Mn}_{x} \mathrm{Si}_{1-x}\right)$ is studied by X-ray absorption spectroscopy at the Mn $L_{3,2}$ edges for $x=0.005-0.18$. Except the $x=0.005$ sample, which shows a slight signature of $\mathrm{Mn}^{2+}$ atomic multiplets associated with a local Mn moment, all samples have broad and featureless $L_{3,2}$ absorption peaks, corresponding to an itinerant state for all $3 d$ electrons. The broad X-ray absorption spectra exclude the possibility of a localized $3 d$ moment and explain the unexpectedly quenched Mn moment in this magnetically-doped amorphous semiconductor. Such a fully delocalized $d$ state of Mn dopant in Si has not been previously suggested.
\end{abstract}

Mn is widely used as a magnetic dopant in diluted magnetic semiconductor (DMS) systems. The value of the Mn moment changes in different matrices due to different local environments. For $\mathrm{Mn}$ in crystalline Si (c$\mathrm{Si}$ ), both mean-field theory (Zener model) [1] and firstprinciples calculations $[2,3]$ predict the presence of large Mn moments $\left(\geq 3 \mu_{B}\right)$ due to the partially-filled localized $3 d$ levels. Experimentally, ferromagnetism is reported in Mn-doped crystalline group-IV semiconductors [4, 5]. However, homogeneous doping of $\mathrm{Mn}$ is difficult, largely due to the extremely low solubility of $\operatorname{Mn}\left(x \sim 10^{-7}\right)$ in crystalline group-IV semiconductors near room temperature [6]. These issues were addressed by preparing Mndoped $\mathrm{Si}$ in the amorphous form, enabling the study of the intrinsic Mn moment in the amorphous $\mathrm{Si}(a-\mathrm{Si})$ environment. Surprisingly, a quenched local Mn moment was observed by magnetic measurements [7].

Ideally transition metal doped ferromagnetic semiconductors rely on robust local moments associated with partially occupied $3 d$ levels, which align via coupling through a low-density carrier system [8]. Magnetization studies on Mn-doped $a$-Si $\left(a-\mathrm{Mn}_{x} \mathrm{Si}_{1-x}\right)$ have shown that $\mathrm{Mn}$ in $a$-Si has an unexpectedly small magnetization for a wide range of $x=0.005-0.18$. Even for the most dilute sample $(x=0.005)$, the majority $(\sim 65 \%)$ of the $\mathrm{Mn}$ atoms are magnetically silent [7]. This low magnetization can be a result of Mn low spin states, antiferromagnetic clusters, or overlapping of $3 d$ atomic orbitals resulting in a nonmagnetic band. With magnetic measurements alone, it is hard to distinguish between these alternative possible explanations.

In this Letter, X-ray absorption spectroscopy (XAS) of the Mn $L$ edges is used to probe the $3 d$ states of Mn in $a$-Si by measuring the transition from occupied $2 p$ core levels to the unoccupied $3 d$ states. Since the magnetic states are closely related to the electronic structure, the local $3 d$ moment can be deduced and compared to magnetization measurements. The measured Mn $L$ edges have

*Electronic address: li_zeng@berkeley.edu a very broad absorption feature, even at very dilute Mn concentration (as low as $x=0.005$ ). The XAS results conclusively show that the $d$-levels are fully extended into bands for doping concentrations $x$ greater than $10^{-3}$ in $a$-Si, even for samples deep on the insulating side of the insulator-metal $(I-M)$ transition.

The $a-\mathrm{Mn}_{x} \mathrm{Si}_{1-x}$ thin film samples were prepared by $e$-beam coevaporation of $\mathrm{Mn}$ and $\mathrm{Si}$ sources under ultrahigh vacuum conditions. Detailed information can be found elsewhere [7]. X-ray absorption (XA) and X-ray magnetic circular dichroism (XMCD) spectra at the Mn $L$ edges were taken at the Advanced Light Source (ALS) beam line 6.3.1, in total electron yield (TEY) mode. All measured thin film samples, including a control sample of a pure Mn metal film, were capped with $2 \sim 4 \mathrm{~nm} \mathrm{Al}$ and stored in a vacuum desiccator to avoid oxidation. Commercially available $\mathrm{Mn}$ oxide powders with different Mn valences $\left(\mathrm{MnO}, \mathrm{Mn}_{2} \mathrm{O}_{3}\right.$ and $\mathrm{MnO}_{2}$, corresponding to $d^{5}, d^{4}$ and $d^{3}$ configurations) were used as references.

Figure 1 shows Mn $L_{3,2}$ spectra for all samples as well as a simulated $\mathrm{Mn}^{2+}$ spectrum. The atomic multiplet features of the $\mathrm{Mn}$ ion in different oxides can be well resolved. Spectra of the oxides are consistent with standard atomic multiplet simulations [9]. We use the main $\mathrm{Mn}^{2+} L_{3}$ absorption peak as our energy reference which occurs at $639.0 \mathrm{eV}$ in our data [Fig. $1(\mathrm{~g})$; the feature at $\sim 641.0 \mathrm{eV}$ comes from $\mathrm{Mn}_{2} \mathrm{O}_{3}$ impurities in the $\mathrm{MnO}$ powder [10]]. The XA spectra of $a-\mathrm{Mn}_{x} \mathrm{Si}_{1-x}$ [Fig. 1(a)(d)] have in general, very broad $L_{3}$ and $L_{2}$ peaks and no XMCD signal is observed in applied external fields up to $0.2 \mathrm{~T}$ at $T=8 \mathrm{~K}$, consistent with the lack of magnetization in ref. 7 . For $x \geq 0.04$, the spectra scale very well with $x$. The spectra of the sputtered Mn metal film and $a-\mathrm{Mn}_{x} \mathrm{Si}_{1-x}$ both show no distinct atomic multiplets, but they differ from each other in peak positions and peak widths: the main absorption peak of the Mn metal has lower energy (639.4 vs $639.9 \mathrm{eV}$ ) and narrower line width.

Figure 2 shows the detailed analysis of the $L$ edges of $a-\mathrm{Mn}_{x} \mathrm{Si}_{1-x}$. For $x \geq 0.04$, the inset of Fig. 2 shows their collapse onto a single curve after normalizing to the concentration-dependent post-edge steps. The spectrum of the $x=0.005$ sample is normalized by the $L_{3}$ peak 


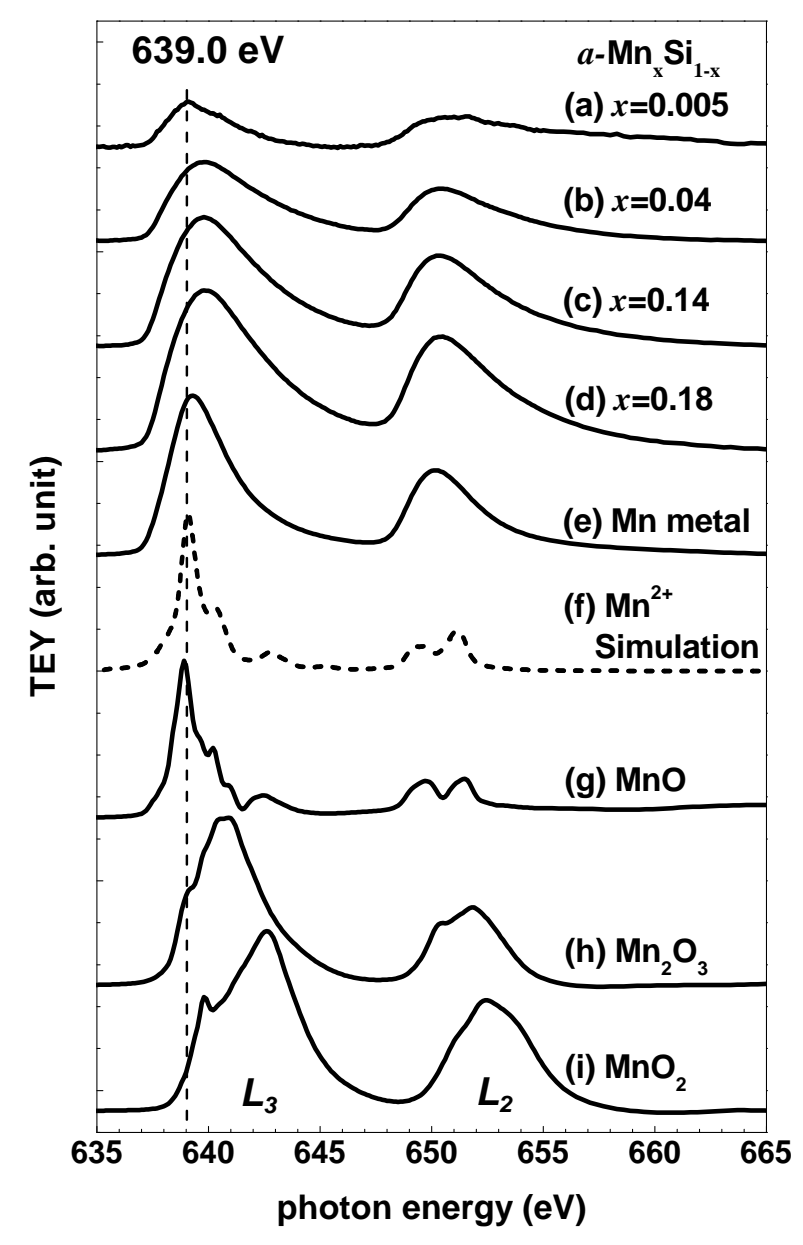

FIG. 1: XAS of Mn $L$ edges for samples and standards. $a-$ $\mathrm{Mn}_{x} \mathrm{Si}_{1-x}$ were prepared by $e$-beam coevaporation. The Mn metal film was prepared by magnetron sputtering at 4 mTorr, all at room temperature. All thin film samples are capped with $2 \sim 4 \mathrm{~nm} \mathrm{Al}$. Mn oxides are commercially available chemicals powders. Compared to Ref. 10, both $\mathrm{MnO}$ and $\mathrm{MnO}_{2}$ samples have an extra feature $\sim 641.0 \mathrm{eV}$, indicating small $\mathrm{Mn}^{3+}$ contribution.

height since the signal/noise ratio is low at/beyond the $L_{2}$-edge energy due to the small $\mathrm{Mn}$ concentration. However, the $x=0.005$ sample obviously exhibits a different line shape at the $L_{3}$ edge with the $L_{3}$ peak at $639.0 \mathrm{eV}$, $0.9 \mathrm{eV}$ lower in energy (with less broadening compared to the $x \geq 0.04$ samples) and aligned with the main $\mathrm{Mn}^{2+}$ $L_{3}$ peak as marked with a dashed line in Fig. 1 .

Besides the $639.0 \mathrm{eV}$ peak, the $L_{3}$ edge of the $x=0.005$ sample also shows a broad shoulder at $\sim 639.9 \mathrm{eV}$, an energy quite similar to the broad peak seen in all samples with $x \geq 0.04$. This strongly suggests that this spectrum consists of a mixture of a $\mathrm{Mn}^{2+}$ multiplet and another broad XA peak centered at $\sim 639.9 \mathrm{eV}$, which is most likely the Mn XA spectra observed in $x \geq 0.04$ sam-

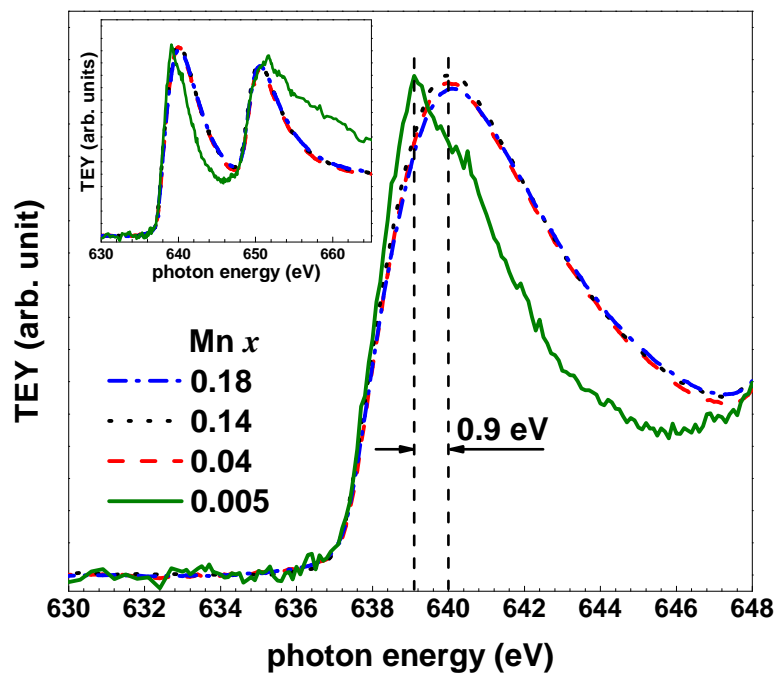

FIG. 2: (color online) $L_{3}$ edges of all $a-\mathrm{Mn}_{x} \mathrm{Si}_{1-x}$ samples. Spectra were normalized to the $L_{3}$ peak height to illuminate the differences in peak positions and peak widths. Inset: normalized spectra (described in the text) show how the overall spectrum shapes of both $L_{3}$ and $L_{2}$ scale with $x$ for $x=0.04$, 0.14 and 0.18 , but do not scale for $x=0.005$.

ples. We test this idea by performing a weighted superposition of the broad spectrum of $x=0.18$ [Fig. 3(a)] and the $\mathrm{Mn}^{2+}$ spectrum [Fig. 3(d)] to match the measured spectrum for the $x=0.005$ sample [Fig. $3(\mathrm{c})]$ at the $L_{3}$ edg. The simulated spectrum [Fig. 3(b)] reproduces the features of the $x=0.005$ sample's spectrum well. The broad absorption peak found in high Mn doping samples develops even at $\mathrm{Mn}$ concentration as low as $x=0.005$, with a significant fraction $(>60 \%)$ of the incorporated $\mathrm{Mn}$, which is reminiscent of the nonmagnetic Mn portion $(\sim 65 \%)$ found in a similar low Mn concentration in the previous magnetization study. These results link the broad XA spectra with the nonmagnetic Mn state. The consistency between the XA data and the magnetization results strengthens the two-state model we proposed for $a-\mathrm{Mn}_{x} \mathrm{Si}_{1-x}[7]$.

The surprising non-magnetic states seen in $a$ $\mathrm{Mn}_{x} \mathrm{Si}_{1-x}$ thus seem to be associated with the broad XA spectra seen here and strongly argues against Mn ions with a low spin state, or AFM coupled Mn moments, because in either case, the XA spectra would have shown multiplet structures. Moreover, large crystal field (CF) splitting is a prerequisite for low spin states, but CF splitting is a small effect in $\mathrm{Si}$ as stated by the Ludwig-Woodbury (LW) model [11]. Disorder in $a$-Si would introduce randomness in the $\mathrm{CF}$ strength. We therefore performed multiplet simulations with varying $\mathrm{CF}$ strengths. The CF of Mn oxides was used as an upper bound of $\mathrm{CF}$ strength in $\mathrm{Si}$, which is found to be $\leq 2$ 


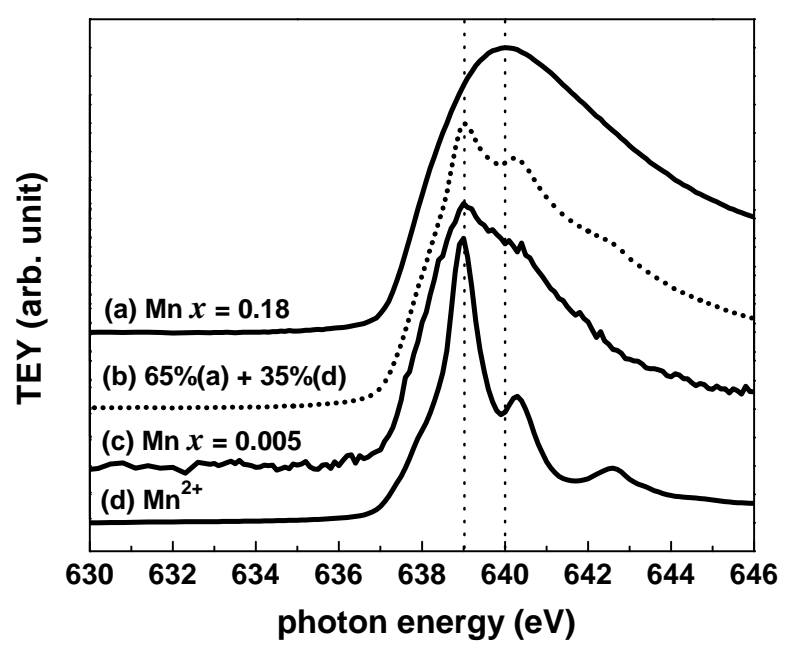

FIG. 3: Demonstration of the existence of mixed states in the $x=0.005$ sample [curve (c)] by superposition of the calculated $L_{3}$ edge of $\mathrm{Mn}^{2+}$ [curve (d)] and of the $x=0.18$ sample [curve (a)]. The dash-dotted curve (b) is the best match to curve (c). The uncertainty of the spectral weight of curve (a) and (d) is $\sim 10 \%$.

$\mathrm{eV}$ (for $\mathrm{Mn}^{4+}$ in $\mathrm{MnO}_{2}$ ) according to our simulations. Varying $\mathrm{CF}$ between 0 and $2 \mathrm{eV}$ produces small effects $(\sim 0.5 \mathrm{eV}$ shift $)$ on the main $L$ peak. Thus even if a random distribution of $\mathrm{CF}$ strengths exists due to disorder, linear combinations of the corresponding weighted spectra would not yield the observed smooth and broad XA peaks (FWHM $\sim 5 \mathrm{eV})$.

Mixed Mn valence states can lead to significant XA broadening, such as found in (La, Ca) $\mathrm{MnO}_{3}$ (LCMO) and $\mathrm{MnSi}$ crystalline compounds. In LCMO, the $L$-edge broadening is due to the co-existence of the $\mathrm{Mn}^{3+}$ and $\mathrm{Mn}^{4+}$ sites [12], and in crystalline $\mathrm{MnSi}$, it is proposed to be the mixed-valence ground state which consists of different $d$ multiplets [13]. However, the underlying features from the atomic multiplets are still clearly visible in those spectra.

In a $p$ - $d$ hybridization scenario, as for the well-studied FM Mn doped III-V DMS systems, multiplet structures were compressed and shifted due to the charge transfer effect and screening [10]. This leads to narrower $L$ peaks and a peak shift of $0.5 \mathrm{eV}$ to lower energy [14, 15], in contrast to what we have observed for $a-\mathrm{Mn}_{x} \mathrm{Si}_{1-x}$ samples, where peaks are broader and shifted $0.9 \mathrm{eV}$ higher in energy. Covalency of Mn-Si bonds is expected to be even stronger than that of Mn-As bonds, and thus cannot account for the observed XA spectra broadening and shift. Moreover, hybridized but sill localized $3 d$ levels should still possess large local Mn moments, which would have been detected by magnetization measurements [15].

We propose instead that the smooth broad $L$ edges of $a-\mathrm{Mn}_{x} \mathrm{Si}_{1-x}$ are most likely due to the formation of an itinerant impurity band of the $3 d$ electrons. Mn metallic clusters are excluded by previous materials characterization [7]. The atomic density obtained from Rutherford backscattering and film thickness measurements increases linearly with Mn doping $x$, suggesting that Mn are at interstitial-like positions with high $\mathrm{Si}$ coordination numbers, as in other crystalline MnSi silicides [13, 16]. The Mn centers and Mn-Si covalent bonds can lead to delocalized states and the formation of an itinerant impurity band. Moments associated with metallic bands are usually small, if present at all, such as in the $\mathrm{Mn}_{4} \mathrm{Si}_{7}$ silicide and the itinerant helimagnet MnSi $[13,17]$. The existence of a well defined band with the electrons being localized due to disorder in these insulating samples (defined as vanishing conductivity at $T \rightarrow 0 \mathrm{~K}$ in $I-M$ transition physics. $x=0.005$ and 0.04 samples are on the insulating side) is consistent with Anderson localization theory [18]. Furthermore the formation of a band by the Mn $3 d$ electrons, perhaps as a hybridization with Si $s$ or $p$ electrons, is possible without long-range order and explains the broad shape of the XA spectra. Also the evidence of a a well defined band in this system can be viewed as a precursor to the MnSi helimagnet with the disorder breaking the long-range order needed for a true magnetic ground state.

In conclusion, we have demonstrated that the XAS of $a-\mathrm{Mn}_{x} \mathrm{Si}_{1-x}$ for $x=0.005-0.18$ can be modeled by a superposition of two distinct electronic states which are associated with a small fraction of $\mathrm{Mn}^{2+}$ magnetic states and a dominant Mn nonmagnetic state, in good agreement with previous magnetization data. The $L$ edges corresponding to the nonmagnetic states have very broad and smooth absorption peaks, which argues against the existence of Mn low spin states and AFM clusters. Instead, the broad XA spectra are evidence of a fully delocalized $d$ state and the formation of impurity bands, which explain the absence of magnetic $\mathrm{Mn}$ in the $a$-Si matrix previously observed.

We thank E. Cruz and D. R. Queen for assistance. Synthesis was supported by NSF DMR-0505524 and measurements were supported by DOE Division of Materials Sciences and Engineering under Contract No. DE-AC02$05 \mathrm{CH} 11231$.
[1] T. Dietl, H. Ohno, F. Matsukura, J. Cibert, and D. Ferrand, Science 287, 1019 (1998).

[2] A. Stroppa, S. Picozzi, A. Continenza, and A. J. Freeman, Phys. Rev. B 68, 155203 (2003).
[3] F. Bernardini, S. Picozzi, and A. Continenza, Appl. Phys. Lett. 84, 2289 (2004).

[4] Y. D. Park, A. T. Hanbicki, S. C. Erwin, C. S. Hellberg, J. M. Sullivan, J. E. Mattson, T. F. Ambrose, A. Wilson, 
G. Spanos, and B. T. Jonker, Science 295, 651 (2002).

[5] M. Bolduc, C. Awo-Affouda, A. Stollenwerk, M. B. Huang, F. G. Ramos, G. Agnello, and V. P. LaBella, Phys. Rev. B 71, 33302 (2005).

[6] E. R. Weber, Appl. Phys. A 30, 1 (1983).

[7] L. Zeng, E. Helgren, M. Rahimi, F. Hellman, R. Islam, B. J. Wilkens, R. J. Culbertson, and D. J. Smith, Phys. Rev. B 77, 073306 (2008).

[8] A. H. MacDonald, P. Schiffer, and N. Samarth, Nature Mat. 4, 195 (2005).

[9] F. M. F. de Groot, Chemical Reviews 101, 1779 (2001).

[10] F. M. F. de Groot, J. Electron Spectrosc. Relat. Phenom. 67, 529 (1994).

[11] G. W. Ludwig and H. H. Woodbury, Phys. Rev. Lett. 5, 98 (1960).

[12] S. Valencia, A. Gaupp, W. Gudat, L. Abad, L. Balcells, A. Cavallaro, B. Martínez, and F. J. Palomares, Phys. Rev. B 73, 104402 (2006).
[13] F. Carbone, M. Zangrando, A. Brinkman, A. Nicolaou, F. Bondino, E. Magnano, A. A. Nugroho, F. Parmigiani, T. Jarlborg, and D. van der Marel, Phys. Rev. B 73, 085114 (2006).

[14] K. W. Edmonds, N. R. S. Farley, T. K. Johal, G. van der Laan, R. P. Campion, B. L. Gallagher, and C. T. Foxon, Phys. Rev. B 71, 064418 (2005).

[15] P. R. Stone, M. A. Scarpulla, R. Farshchi, I. D. Sharp, D. D. Haller, O. D. Dubon, K. M. Yu, J. W. Beeman, E. Arenholz, J. D. Denlinger, et al., Appl. Phys. Lett. 89, 012504 (2006).

[16] H. W. Knott, M. H. Mueller, and L. Heaton, Acta Cryst. 23, 549 (1967).

[17] U. Gottlieb, A. Sulpice, B. Lambert-Andron, and O. Laborde, J. Alloys Comp. 361, 13 (2003).

[18] P. W. Anderson, Phys. Rev. 124, 41 (1961). 